\title{
5G-Enabled Tactile Internet for smart cities: vision, recent developments, and challenges
}

\author{
Tanweer Alam \\ Department of Computer Science, Faculty of Computer and Information Systems, \\ Islamic University of Madinah, Saudi Arabia \\ Email: tanweer03@iu.edu.sa
}

\begin{abstract}
The Tactile Internet (TI) is an emerging technology next to the Internet of Things (IoT). It is a revolution to develop smart cities, communities, and cultures in the future. This technology will allow the real-time interaction between humans and machines as well as machine-to-machine with the $1 \mathrm{~ms}$ challenge to achieve in round trip latency. The term TI is defined by the International Telecommunication Union (ITU) in August 2014. The TI provides a fast, reliable, secure and available internet network that is the requirements of the smart cities in 5G. Tactile internet can develop the part of the world where the machines are strong, and humans are weak. It increases the power of machines so that the value of human power will increase automatically. In this framework, we have presented the idea of tactile internet for the next generation of smart cities. This research will provide a highperformance reliable framework for the internet of smart devices to communicate with each other in a real-time (1ms round trip) using IEEE 1918.1 standard. The objective of this research is expected to bring a new dimension in the research of smart cities.
\end{abstract}

\author{
Keywords: \\ Tactile Internet (TI) \\ Internet of Things (IoT) \\ IEEE 1918.1 standard \\ Ultra-Low latency \\ Smart Cities
}

\section{Introduction}

The proposed research entitled "Tactile Internet-based reliable communication framework for Smart cities in $5 \mathrm{G}^{\prime}$ " is a step forward in wireless networking and IoT where we propose a new reliable framework based on Tactile Internet.

Wireless communication is the key to the Internet of things and Tactile Internet. It is expected to exceed 50 billion connected devices by 2020 and most of these nodes cannot be connected by wireline. In order to enable critical applications such as smart factories or smart buildings, the networking protocols must deal with the non-deterministic nature of wireless links. In the 5th generation communication system, secure and reliable data packets will rely on the network with high availability and low latency [1].

$5 \mathrm{G}$ enables the dynamic control of nodes and low latency [2]. The ultra-reliability feature is an interface working with high availability and low latency in Tactile Internet that brings in fifthgeneration networks [3]. The tactile internet will act in the area of societies so that it required an ultra-reliability feature to empower the peoples as well as machines for collaborating with their neighbors [4]. The connections among ultra-reliability networks are an extremely hard situation to keep low packets corruption [5].

The tactile internet will provide a platform for measuring, controlling, monitoring and scaling the smart objects in reality or virtually in the smart cities [6]. The ultra-low latency, reliability, and availability in controlling are the main features of tactile internet that make it advanced in 5G [7].

Because of these features, the internet wants to move from mobile to Tactile Internet. The proposed research work is an enhancement and implementation of a reliable framework based on Tactile Internet emerging technology next to the internet of things [8], [9]. The research outcome is to establish a new reliable framework for communication among humans to machine and machine to machine in the future smart cities [10]. The proposed research uses the correct and efficient simulation of the desired study and can be implemented in a framework of smart cities. In the 
future, researchers can enhance this research and implement it on the internet of everything framework [11]. Figure 1 shows the applications of 5G.

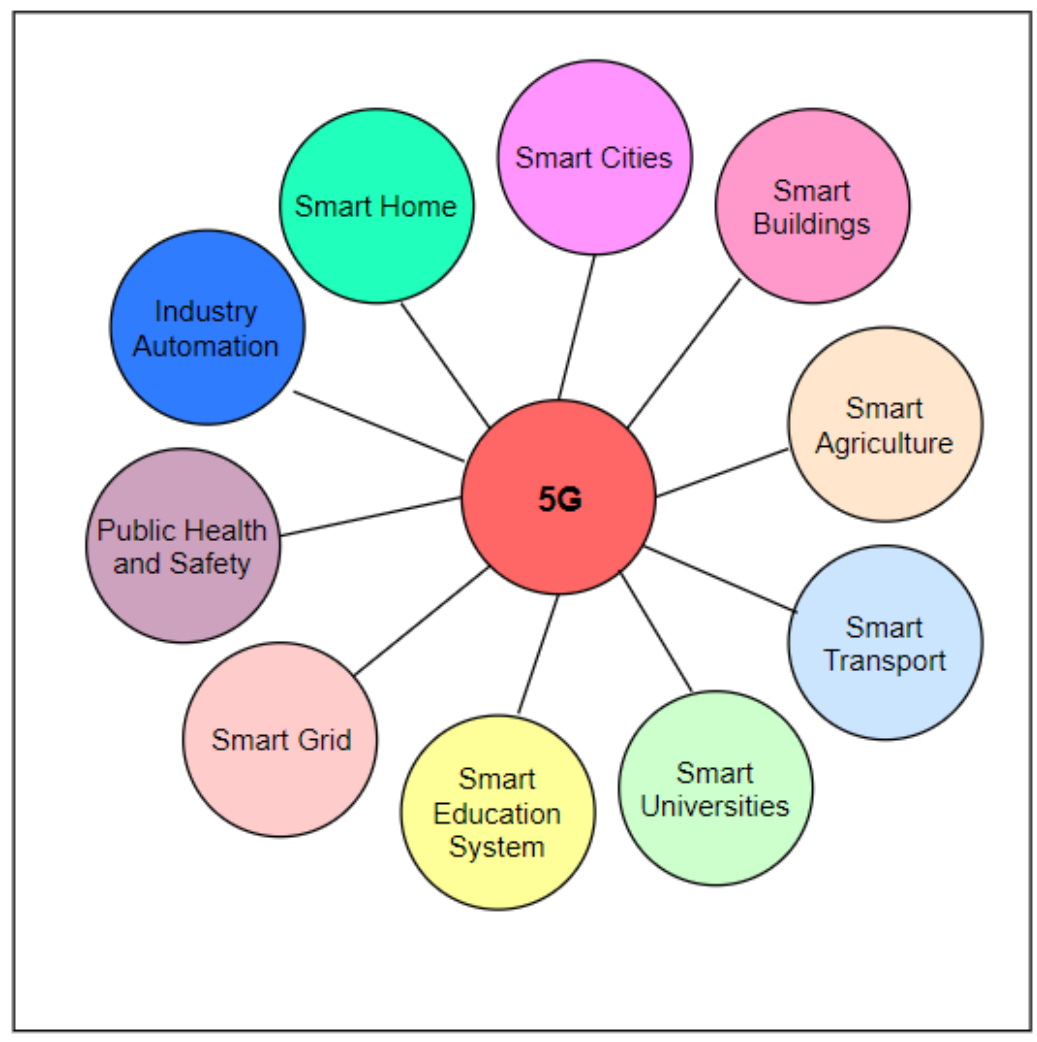

Fig. 1. 5G Applications

The objective of this research is to create a new reliable communication framework for smart cities using the Tactile Internet the next revolution of the internet of things. This research is based on low-latency, ultra-high availability and high-performance concepts of Tactile Internet. The framework provides QoS through reducing the latency ( $1 \mathrm{~ms}$ in the round trip) also the variety of the number of smart devices. In this research, I consider the idle state in order to make our examination more efficient, at that point the general execution regarding the overall performance of the framework is evaluated. The framework will monitor and analyze the real-time data collected from the network and then taking the action.

The research is primarily focused on the next-generation Internet for smart cities. It enables smart devices to communicate with another device among the internet of smart devices using fast, reliable and secure tactile internet. The proposed framework for communication will access the internet of smart devices. The results of the proposed research will be compared with the previous study in the same area.

The rest of the paper is organized as follows. Section II represents the related works, Section III represents the research methodologies, Section IV represents the applications of the Tactile Internet, Section V represents the recent developments, Section VI shows the challenges and Section VII represents the results and discussion and section VIII shows the conclusion.

\section{Related Works}

In 1991, Theodore S. Rappaport published an article entitles "The wireless revolution", in this paper he presented the wireless communications is the emerging technology as a key for communication among humans as well as devices [12]. In the med of 2006, Amazon achieved a prominent milestone by testing elastic computing cloud (EC 2) which initialized the spark of cloud computing in it. However, the term cloud computing has not coned until March 2007 [13]. The following year brought even more rapid development of the newly emerged paradigm. 
Furthermore, cloud computing infrastructure services have widened to include (SaaS) software as a service [14]. In the mid of 2012, the oracle cloud has been introduced, where it supports different deployment models. It is provisioned as the first unified collection of its solutions which is under continues developments. Nowadays, typing cloud computing in any search engine will result in a tremendous result. For example, it would result in more than 139,000,000 matches on Google.

Table.1: Comparison of peak data rate and latency [13]

\begin{tabular}{|c|c|c|}
\hline Technology & Peak Data Rate & Latency \\
\hline GPRS & $114 \mathrm{kbps}$ & $\sim 500 \mathrm{~ms}$ \\
\hline EDGE & $236.8 \mathrm{kbps}$ & $\sim 250 \mathrm{~ms}$ \\
\hline W-CDMA & $384 \mathrm{kbps}$ & $\sim 200 \mathrm{~ms}$ \\
\hline HSPA & $2 \mathrm{Mbps}$ & $\sim 150 \mathrm{~ms}$ \\
\hline HSPA & $42 \mathrm{Mbps}$ & $\sim 70 \mathrm{~ms}$ \\
\hline LTE & $300 \mathrm{Mbps}$ & $\sim 30 \mathrm{~ms}$ \\
\hline LTE-A & $1 \mathrm{Gbps}$ & $\sim 20 \mathrm{~ms}$ \\
\hline
\end{tabular}

In 2019, Ishan Budhiraja, et. al. published a paper [15], In this paper, the authors have presented the tactile internet for smart communities in 5G. They summarize the use of non-orthogonal multiple access protocols in 5G. In the technical report [16], the authors represented the tactile internet as the next revolution after the Internet of things.

In the article [17], the cloud-based queuing model is explained for the tactile internet. In the article [18], the author discussed the haptic communication system. In the article [19], the authors have enabled the tactile internet for ultra-reliability and fast response time $(<1 \mathrm{~ms})$.

In the paper [20], the authors present a review on tactile internet for industries. It represents the role of tactile internet in the future industries. In the thesis [21], the author explored the challenges and standards for the tactile internet in $5 \mathrm{G}$.

In [22], the 5G-based Tactile internet framework is designed. Very few articles are written on Tactile internet. The previous studies showed us the role, use of tactile internet in the $5^{\text {th }}$ generation.

\section{Research Methodology}

The smart devices are increasing exponentially day by day in the whole world [23]. They provide many more facilities to the end-users and also attach to their daily life [24]. Smart devices can connect to the internet easily for sending and receiving data within the network [25].

The smart devices are not just smartphones [26], it may be the smart refrigerator, Smart home automation entry point, smart air conditioners, smart hubs, Smart thermostat, Color-changing smart LEDs, Smart Watches and Smart Tablets, etc. in the internet of things framework, they are connected to each other through the internet [27], [28], [29]. Figure 2 represents the human reaction time.

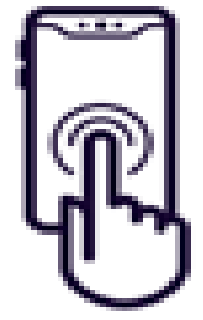

15

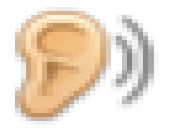

$100 \mathrm{~ms}$

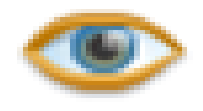

$10 \mathrm{~ms}$

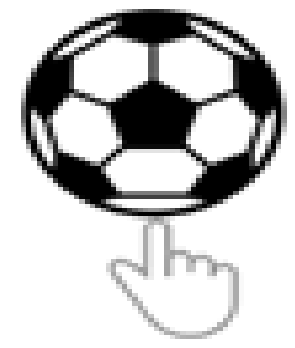

$1 \mathrm{~ms}$

Fig. 2: Human reaction time 
Table 2. IoT Devices installed category and year wise (in Millions)

\begin{tabular}{|c|c|c|c|c|}
\hline Category & 2016 & 2017 & 2018 & 2020 \\
\hline IoT Devices & 3963.0 & 5244.3 & 7036.3 & 12863.0 \\
\hline Business: Across Industries & 1102.1 & 1501.0 & 2132.6 & 4381.4 \\
\hline Business: Vertical specific & 1316.6 & 1635.4 & 2027.7 & 3171.0 \\
\hline Total & 6381.8 & 8380.6 & 11196.6 & 20415.4 \\
\hline
\end{tabular}

The proposed research plan builds research on extending the performance of communication in the internet of things using tactile internet. The transfer data from one configuration to another using a wireless network starts from 1973 in the form of the packets radio network. They were able to communicate with other same configuration devices. Recent work is continuing a project called the Serval Project. It provides networks facility to android devices for communication in an infrastructure-less network [30], [31]. Whereas our research is concerned about high-performance communication on the internet of smart devices for smart cities.

The main contribution of this research is the creation of a reliable communication framework and provide secure, reliable and fast communication using Tactile Internet among the internet of smart devices. The previous studies have been focused on the creation and optimization of the framework for communication, but such research doesn't perform the full framework for secure and reliable communication among the internet of smart devices for smart cities. Figure 3 shows the tactile internet in $5 \mathrm{G}$.

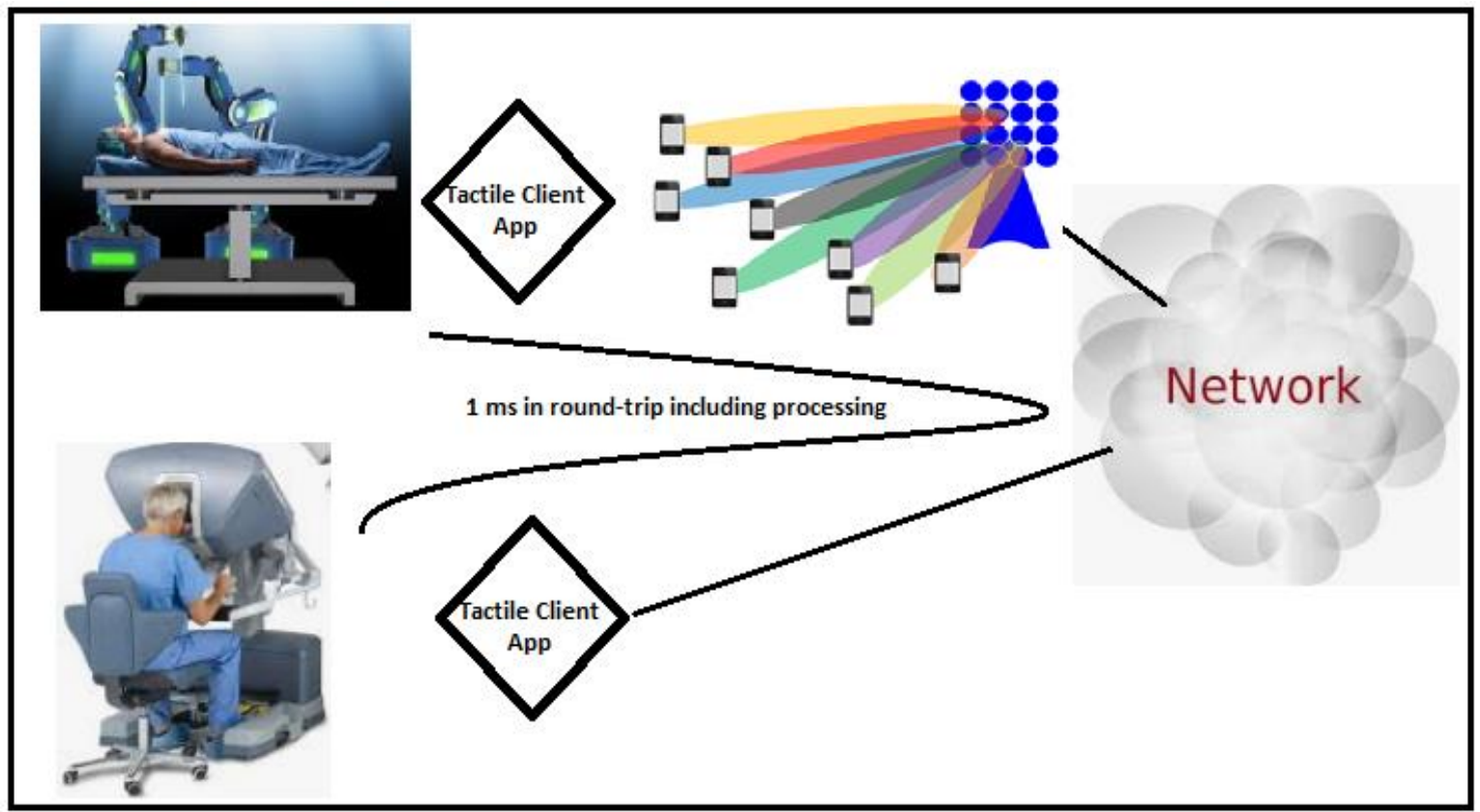

Fig. 3: 5G enabled Tactile Internet

\section{Tactile Internet Framework}

The Tactile Internet necessitates the optimum turnaround time, accessibility, reliability, and security. Such goals can also be accomplished through the construction of a distributed service model. There is a need for ultra-low end-to-end latency to encourage tactile apps to remain regional, near to subscribers. Figure 4 represents the tactile internet working process. 


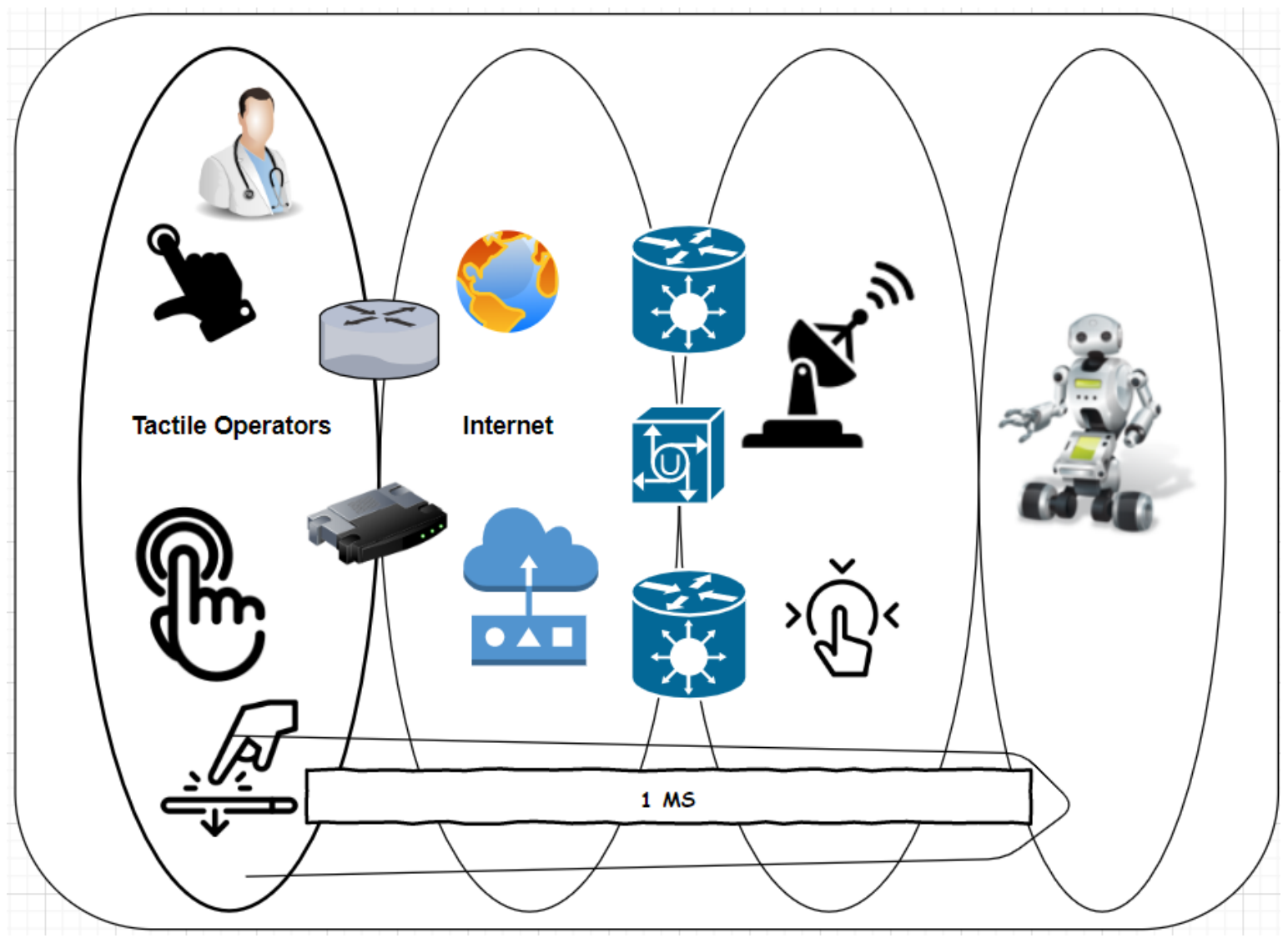

Fig. 4: Tactile Internet framework

\section{Applications}

Numerous apps in several diverse areas might be produced through the tactile Internet, such as the following.

\section{A. Health apps}

The Tactile Internet is predicted to facilitate public health and safety via the implementation of new apps and services that do not endorse conventional systems. Such apps involve remote rehabilitation, remote treatment, and remote surgical intervention. Such apps would make premium medical training and healthcare professionals accessible everywhere and break the restrictions of place and time [32].

\section{B. Vehicle apps}

The Tactile Internet is supposed to support in the management of road transport via traffic detectors as well as driver-assist schemes. The Tactile Internet would enable and to provide the vehicle-to-vehicle connectivity function as well as the vehicle-to-vehicle highway communication networks [32].

\section{Apps for industrial automation System}

The closed-loop touch with the reality circuit for most industrial automated machines necessitates an end-to-end latency per sensor of $1 \mathrm{~ms}$. It could be accomplished by implies of a tactile Internet structure, as well as the present connected model could be transformed into wireless or enhanced infrastructures. It will allow multiple automated systems or improve the efficiency of the current approach [32]. 


\section{Smart Grid Apps}

An intelligent network is built to distribute energy efficiently generated and with the necessary reliability of the power consumption. Smart grid networks effectively control the activity of both power generators and power grids. Also, user usage or tariffs are controlled and monitored by smart grid technologies. These systems, therefore, need ultra-reliability and low-latency communications networks (e.g. the recommended end-to-end latency of the symmetric co-phase of energy supplies is $1 \mathrm{~ms}$ ) to transmit information over the Internet. The Tactile Internet would defend such structures by accomplishing an end-to-end latency of $1 \mathrm{~ms}$ with ultra-high reliability [32].

\section{E. Other Apps}

It is expected that the Tactile Internet will have apps in many other important areas such as education, community, professional gameplay, and surveillance drones, etc. The Tactile Internet would help to develop apps that could support children and individuals with disabilities in enhancing their learning skills, recovering from injuries or disability skill sets [32].

\section{Recent Developments}

The following are the recent developments in the area of Tactile Internet.

\section{A. Deep Learning-based reliable connectivity}

The Tactile Internet requires ultra-reliable connectivity through huge IoT gadgets. The grantfree non-orthogonal multi-access (NOMA) utilizes the combined advantage of grant-free access and non-orthogonal communications to obtain lower latency significant accessibility. Moreover, this suffered from lowered accuracy due to random intrusion. They formulated a variation optimization challenge to enhance the performance of the grant-free access. The deep learning was used to parameterize unsolvable variational functionality with a designed deep neural network [33].

\section{B. Tactile Robots}

This new frontier of an interactive coexistence between humans and robots constructs on various new technologies in automation, multi-modal teleoperation, smartwatches, distributed computation, and mobile technologies [34].

\section{Tactile Internet Architecture for Smart City}

In order to completely integrate tactile technology with smart cities, the new QoE-driven Tactile Internet framework for smart cities including five layers: sensing layer, transmission layer, processing layer, computing layer, and application layer was designed. Specifically, the strategies represented in each layer of this architectural design comply with the criteria of low latency, high reliability, and high user expertise. Within this framework, they are designing a fast and reliable QoE management platform focused on a large training system [35].

\section{Challenges}

Clearly, allowing a 1-ms round-trip latency is a major challenge in Tactile Internet. Physical transmission might have very small packets to allow $100 \mu$ s of one-way physical layer transmission

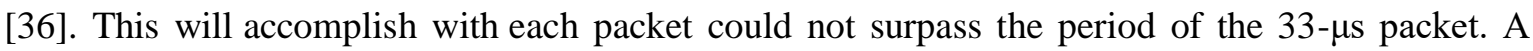
possible explanation for this is the structural extra latency that requires to be implemented by encrypting the packet on the transmitter and detecting and decryption it on the transmitter. It restricts the packet size to less than one-third of the targeted latency. It clearly shows that the synchronization used throughout recent LTE wireless networks is not a feasible solution [37]. Figure 5 shows the roadmap of the wireless network from 1995 to 2030. 


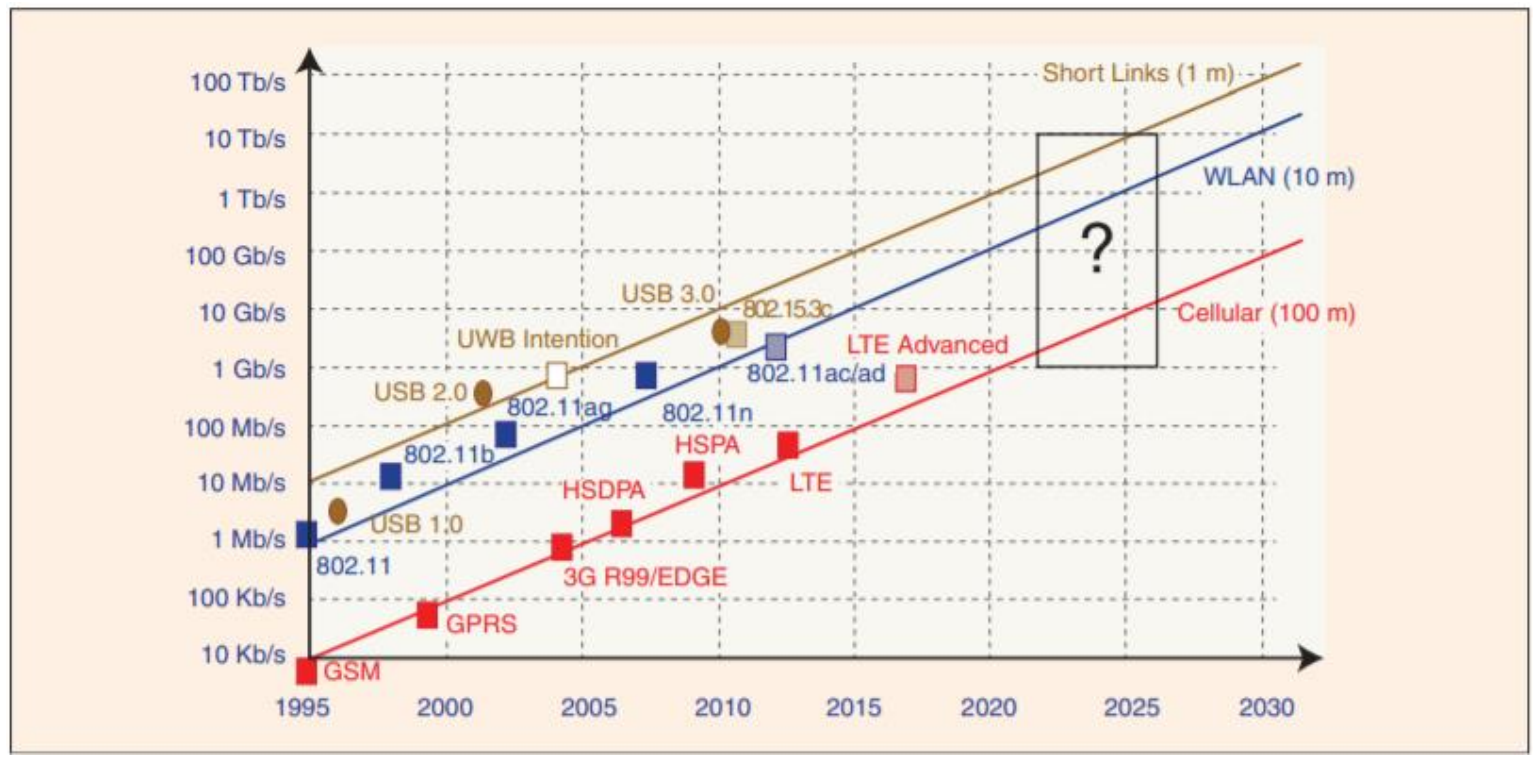

Fig 5. The Wireless roadmap from 1995-2030 [36]

\section{Discussion}

By moving to $1 \mathrm{~ms}$ round-trip latency together with carrier-grade robustness and accessibility, a new innovation that enables unparalleled mobile apps became feasible. Such systems are called the Tactile Internet as this is the standard latency communication needed for tactile operation and control of real and virtual objects without the development of cybersickness. It will revolutionize education, accessibility and traffic, healthcare, sports, culture, gaming, and the smart grid, just to mention a few of the segments that can be used. The Tactile Internet must radically reshape our culture.

\section{Conclusion}

The main contribution of this research is designing a framework for ultra-reliable, low latency and high availability communication on the Internet of smart devices for future smart cities using the Tactile Internet. The proposed framework is specifically appropriate for applications in which data is periodically transmitted on the internet of smart devices environment. In these applications, on one hand, packets are being produced based on a certain period of time pattern. On the other hand, the service time is always a random variable with the general distribution. Therefore, service time might temporarily exceed the period time which, as an inevitable consequence some packets might encounter a busy channel and be dropped. We solve this problem by proposing the new communication framework. We demonstrate that the proposed reliable framework, not only increases the throughput but also the direct connection between the generation (sensors) and communication packet systems are eliminated which makes the system far more stable. Moreover, in order to enhance the proposed model, we have employed a retransmission scheme, variable packet length, and saturated traffic condition. The solution to this research is summarized as follows. The implementation of the proposed framework for communication among the internet of smart devices in $5 \mathrm{G}$ will be programmed to execute on to the internet of things using Tactile Internet concepts. The idea will focus on three main concepts, these concepts are Reliability, Security, and availability. The proposed study supports wireless networking technology to establish a reliable framework among the internet of devices for smart cities.

\section{References}

[1] Li, Shancang, Li Da Xu, and Shanshan Zhao. "The internet of things: a survey." Information Systems Frontiers 17.2 (2015): 243-259. Alam, Tanweer. "Middleware Implementation in Cloud-MANET Mobility Model for Internet of Smart Devices", International Journal of Computer Science and Network Security, 17(5), 2017. Pp. 86-94 
[2] Alam T, Benaida M. "CICS: Cloud-Internet Communication Security Framework for the Internet of Smart Devices”, International Journal of Interactive Mobile Technologies (iJIM). 2018 Nov 1;12(6):7484. DOI: https://doi.org/10.3991/ijim.v12i6.6776

[3] Tanweer Alam, "Blockchain and its Role in the Internet of Things (IoT)", International Journal of Scientific Research in Computer Science, Engineering and Information Technology, vol. 5(1), pp. 151157, 2019. DOI: https://doi.org/10.32628/CSEIT195137

[4] Tanweer Alam, Baha Rababah, "Convergence of MANET in Communication among Smart Devices in IoT", International Journal of Wireless and Microwave Technologies(IJWMT), Vol.9, No.2, pp. 1-10, 2019. DOI: https://doi.org/10.5815/ijwmt.2019.02.01

[5] Tanweer Alam, "IoT-Fog: A Communication Framework using Blockchain in the Internet of Things", International Journal of Recent Technology and Engineering (IJRTE), Volume-7, Issue-6, 2019.

[6] Alam, Tanweer, and Mohammed Aljohani. "Design and implementation of an Ad Hoc Network among Android smart devices." In Green Computing and Internet of Things (ICGCIoT), 2015 International Conference on, pp. 1322-1327. IEEE, 2015. DOI: https://doi.org/10.1109/ICGCIoT.2015.7380671

[7] Alam, Tanweer, and Mohammed Aljohani. "An approach to secure communication in mobile ad-hoc networks of Android devices." In 2015 International Conference on Intelligent Informatics and Biomedical Sciences (ICIIBMS), pp. 371-375. IEEE, $2015 . \quad$ DOI: https://doi.org/10.1109/iciibms.2015.7439466

[8] Aljohani, Mohammed, and Tanweer Alam. "An algorithm for accessing traffic database using wireless technologies." In Computational Intelligence and Computing Research (ICCIC), 2015 IEEE International Conference on, pp. 1-4. IEEE, 2015. DOI: https://doi.org/10.1109/iccic.2015.7435818

[9] Alam, Tanweer, and Mohammed Aljohani. "Design a new middleware for communication in ad hoc network of android smart devices." In Proceedings of the Second International Conference on Information and Communication Technology for Competitive Strategies, p. 38. ACM, 2016. DOI: https://doi.org/10.1145/2905055.2905244

[10] Alam, Tanweer. (2018) "A reliable framework for communication in internet of smart devices using IEEE 802.15.4." ARPN Journal of Engineering and Applied Sciences 13(10), 3378-3387.

[11] Tanweer Alam, "A Reliable Communication Framework and Its Use in Internet of Things (IoT)", International Journal of Scientific Research in Computer Science, Engineering and Information Technology (IJSRCSEIT), Volume 3, Issue 5, pp.450-456, May-June.2018 URL: http://ijsrcseit.com/CSEIT1835111.

[12] Rappaport, Theodore S. "The wireless revolution." IEEE Communications Magazine 29, no. 11 (1991): $52-71$.

[13] Garfinkel, Simson. "An evaluation of amazon's grid computing services: EC2, S3, and SQS." (2007).

[14] M. Chowdhury and M. Maier, "Local and Non-Local Human-to-Robot Task Allocation in FiberWireless Multi-Robot Networks,” IEEE Systems Journal, vol. 12, no. 3, pp. 2250-2260, Sept. 2018

[15] Budhiraja I, Tyagi S, Tanwar S, Kumar N, Rodrigues JJ. Tactile Internet for Smart Communities in 5G: An Insight for NOMA-based Solutions. IEEE Transactions on Industrial Informatics. 2019 Jan 14.

[16] Aijaz, Adnan, Mischa Dohler, A. Hamid Aghvami, Vasilis Friderikos, and Magnus Frodigh. "Realizing the tactile internet: Haptic communications over next generation 5G cellular networks." IEEE Wireless Communications 24, no. 2 (2017): 82-89.

[17] Fettweis, Gerhard, and Siavash Alamouti. "5G: Personal mobile internet beyond what cellular did to telephony." IEEE Communications Magazine 52, no. 2 (2014): 140-145.

[18] Fettweis, Gerhard P. "The tactile internet: Applications and challenges." IEEE Vehicular Technology Magazine 9, no. 1 (2014): 64-70. 
[19] Ji, Hyoungju, Sunho Park, Jeongho Yeo, Younsun Kim, Juho Lee, and Byonghyo Shim. "Ultra-reliable and low-latency communications in 5G downlink: Physical layer aspects." IEEE Wireless Communications 25, no. 3 (2018): 124-130.

[20] Aijaz A, Sooriyabandara M. The Tactile Internet for Industries: A Review [35pt]. Proceedings of the IEEE. 2018 Nov 21.

[21] Cakuli, Julian. "Application Scenarios, Research Challenges and Standardization for Tactile Internet." (2017).

[22]Li, Chong, Chih-Ping Li, Kianoush Hosseini, Soo Bum Lee, Jing Jiang, Wanshi Chen, Gavin Horn, Tingfang Ji, John E. Smee, and Junyi Li. "5G-based systems design for tactile Internet." Proceedings of the IEEE 99 (2018): 1-18.

[23] Alam, Tanweer. "Fuzzy control based mobility framework for evaluating mobility models in MANET of smart devices." ARPN Journal of Engineering and Applied Sciences 12, no. 15 (2017): 4526-4538.

[24] Alam, Tanweer, Arun Pratap Srivastava, Sandeep Gupta, and Raj Gaurang Tiwari. "Scanning the Node Using Modified Column Mobility Model." Computer Vision and Information Technology: Advances and Applications 455 (2010).

[25] Alam, Tanweer, Parveen Kumar, and Prabhakar Singh. "SEARCHING MOBILE NODES USING MODIFIED COLUMN MOBILITY MODEL.", International Journal of Computer Science and Mobile Computing, (2014).

[26] Alam, Tanweer, and B. K. Sharma. "A New Optimistic Mobility Model for Mobile Ad Hoc Networks." International Journal of Computer Applications 8.3 (2010): 1-4. DOI: https://doi.org/10.5120/1196-1687

[27] Singh, Parbhakar, Parveen Kumar, and Tanweer Alam. "Generating Different Mobility Scenarios in Ad Hoc Networks.", International Journal of Electronics Communication and Computer Technology, 4(2), 2014

[28] Sharma, Abhilash, Tanweer Alam, and Dimpi Srivastava. "Ad Hoc Network Architecture Based on Mobile Ipv6 Development." Advances in Computer Vision and Information Technology (2008): 224.

[29] Aljohani, Mohammed, and Tanweer Alam. "Real Time Face Detection in Ad Hoc Network of Android Smart Devices", Advances in Computational Intelligence: Proceed-ings of International Conference on Computational Intelligence 2015. Springer Singa-pore, 2017.DOI: https://doi.org/10.1007/978-981-102525-9_24

[30] M. Aljohani and T. Alam, "Design an M-learning framework for smart learning in ad hoc network of Android devices," 2015 IEEE International Conference on Computational Intelligence and Computing Research (ICCIC), Madurai, 2015, pp. 1- 5. DOI: https://doi.org/10.1109/ICCIC.2015.7435817

[31] Alam, T. and Benaida, M., "The Role of Cloud-MANET Framework in the Internet of Things (IoT)". International Journal of Online and Biomedical Engineering (iJOE), 14(12), pp.97-111. DOI: https://doi.org/10.3991/ijoe.v14i12.8338

[32] Ateya, Abdelhamied A., Ammar Muthanna, Anastasia Vybornova, Irina Gudkova, Yuliya Gaidamaka, Abdelrahman Abuarqoub, Abeer D. Algarni, and Andrey Koucheryavy. "Model Mediation to Overcome Light Limitations-Toward a Secure Tactile Internet System." Journal of Sensor and Actuator Networks 8, no. 1 (2019): 6. DOI: https://doi.org/10.3390/jsan8010006

[33] Ye, Neng, Xiangming Li, Hanxiao Yu, Aihua Wang, Wenjia Liu, and Xiaolin Hou. "Deep Learning Aided Grant-Free NOMA Toward Reliable Low-Latency Access in Tactile Internet of Things." IEEE Transactions on Industrial Informatics 15, no. 5 (2019): 2995-3005. DOI: https://doi.org/10.1109/TII.2019.2895086

[34] Haddadin, Sami, Lars Johannsmeier, and Fernando Díaz Ledezma. "Tactile Robots as a Central Embodiment of the Tactile Internet." Proceedings of the IEEE 107, no. 2 (2018): 471-487. DOI: https://doi.org/10.1109/JPROC.2018.2879870 
[35] Wei, Xin, Qi Duan, and Liang Zhou. "A QoE-Driven Tactile Internet Architecture for Smart City." IEEE Network (2019). DOI: https://doi.org/10.1109/MNET.001.1900078

[36] Fettweis, Gerhard P. "The tactile internet: Applications and challenges." IEEE Vehicular Technology Magazine 9, no. 1 (2014): 64-70. DOI: https://doi.org/10.1109/MVT.2013.2295069

[37] Alam, Tanweer. "Tactile Internet and its Contribution in the Development of Smart Cities." arXiv preprint arXiv:1906.08554 (2019). 